Rodríguez-Triana, Z.E. \& Suárez-Ortíz, J.L. (2019). Escuelas familiares. Una experiencia favorecedora para el desarrollo humano. Revista Electrónica Interuniversitaria de Formación del Profesorado, 22(3), 127-138.

DOI: http://dx.doi.org/10.6018/reifop.22.3.390161

\title{
Escuelas familiares. Una experiencia favorecedora para el desarrollo humano
}

Zulema Elisa Rodríguez Triana, Jazmín Lorena Suárez Ortiz

Departamento de Estudios de Familia, Facultad de Ciencias Jurídicas y Sociales, Universidad de Caldas. Manizales, Colombia.

\section{Resumen}

La familia y la escuela son contextos de desarrollo para niños y niñas y, aunque comparten intencionalidades de formación, parecen caminar por senderos diferentes. Las escuelas familiares orientadas desde un enfoque de las capacidades y la corresponsabilidad sobre la base de los niños y niñas como titulares de derechos y actuadas mediante alternativas de formación y del fortalecimiento de la participación de la familia en la escuela son, desde la experiencia que deriva esta reflexión, una estrategia socioeducativa que favorece el desarrollo humano de los actores. Se asume una práctica investigativa construida a partir de la Investigación Acción Participante (IAP) en Manizales, Colombia con el acompañamiento de la Universidad de Caldas.

\section{Palabras clave}

Escuelas familiares; Desarrollo humano, Capacidades; Oportunidades.

\section{Family schools. A favorable experience for human development}

\section{Abstract}

Family and school are developmental contexts for boys and girls and, although they share training intentions, they seem to walk different paths. Family schools oriented from a capacities and co-responsibility approach based on children as holders of rights and acted

\section{Contacto:}

Zulema Elisa Rodríguez Triana, zulema.rodriguez@ucaldas.edu.co 
on through training alternatives and strengthening family participation in school are, from the experience that derived this reflection, a socio-educational strategy that favors the human development of the actors. A research practice built from Participatory Action Research (PAR) is held in Manizales, Colombia with the support of the University of Caldas.

\section{Keywords}

Family schools; Human development, Capacities; Opportunities.

\section{Introducción}

Las familias y la escuela son entornos educativos que favorecen la construcción de conocimientos, la incorporación de hábitos, habilidades y destrezas, al igual que la formación en valores y la construcción de significados. Cada una, desde su lugar y responsabilidad social y de las relaciones que construye con y entre sus integrantes, cumple funciones particulares, pues "ni la familia ni la escuela pueden, por sí solas, afrontar la formación de la niñez y la juventud. Se hace necesario un acercamiento en el que la educación familiar se coloque como posibilidad pedagógica de tal confluencia" (Páez y Pérez, 2018, p. 9).

A este propósito, Lacasa (2008) propone como primer elemento para la definición de la relación, la consideración de la familia y la escuela como comunidades de prácticas que se fundamentan a través de la interacción entre sus integrantes, donde cada uno cumple un rol reconocido y donde se utilizan instrumentos que cumplen determinadas funciones. De igual modo, Rogoff (1997) asume la comunidad de prácticas como un grupo de personas que interactúan y comparten formas de organización, valores y prácticas. La interacción, según la autora, "crea lazos de dependencia y de afecto entre los individuos que se expresan a través de códigos simbólicos, sentimientos y creencias compartidas y una ética de la vida en común" (p. 48).

Las familias definidas por la configuración de relaciones parentales y vinculantes dadas por la intimidad poseen, en palabras de Lacasa (1997), fondos de conocimiento considerados como ese universo de creencias y valores que se encubren detrás de sus prácticas cotidianas y se vinculan con las diferentes actividades y recursos que necesitan para subsistir. Esto quiere decir que en los grupos familiares sus integrantes comparten una historia, configuran motivos e intenciones, despliegan prácticas y discursos, cumplen tareas para sí y para otros y movilizan recursos, capacidades y emociones.

Asimismo, la escuela como posibilidad ha de ser un escenario de mediación cultural donde confluyan procesos de enseñanza y aprendizaje a partir de relaciones vivenciales y democráticas que faciliten a los actores construir significados y constituirse como sujetos autoreferenciales. En otras palabras, siguiendo a Giroux (2006), la escuela es un contexto para la participación democrática de los estudiantes, en la que pueden compartir sus sentimientos, vivencias, apropiar conocimientos -no solo formales- y proyectar su interacción con la sociedad.

La relación familia-escuela debe pensarse en términos de los niños, niñas y adolescentes en su condición de sujetos de derechos y buscar su reconocimiento y la satisfacción de sus necesidades materiales y subjetivas, fundamentadas en una nueva ética para promover su desarrollo. El hecho de comprender a los niños como seres que se desarrollan en la vida 
social gracias a la interacción con las personas que les rodean simboliza que requieren de personas, hombres y mujeres, adultas capaces de acompañarles en su proceso y garantizarles condiciones y calidad de vida acordes a sus requerimientos y capacidades.

A este respecto, Arias (2017) afirma que:

La infancia es un estado del ser humano, no es una condición de dignidad o reconocimiento limitada por estados de incompletud; el niño o la niña son seres completos, dignos, son ciudadanos partícipes desde su condición de ciudadanía, su voz debe ser escuchada como se escucha la voz del adulto. Es recurrente, sin embargo, la pregunta del adulto cuando de derechos de los niños se habla, y ¿dónde están los deberes? Este ejercicio requiere asegurar que, sin derechos, no hay deberes $y$, por otra parte, no se le restituye a nadie los derechos que nunca ha tenido, una ruta de restitución de derechos a una infancia que nunca los ha tenido, no se pierde lo que no ha tenido, ni le devuelven lo que nunca perdió, simplemente no lo tuvo. (p. 132)

Si la intención de la relación entre familia y escuela está a favor de los niños-as en su titularidad de derecho, ha de considerarse el diálogo como mediador de un intercambio comunicativo continuo en el que se reconozca la confianza, la esperanza, la capacidad de escucha y la búsqueda de alternativas para aportar al desarrollo de los sujetos que los une. El diálogo entonces es una relación y la relación implica participación y capacidad de actuación para elegir, decidir e incidir en situaciones que se deben atender de manera compartida.

Así pues, se debe generar un nuevo pacto educativo que articule la acción educativa escolar con la de otros agentes (Tedesco, 1995) como la familia y para ello hay que apelar a estrategias que, como las escuelas familiares, hagan posible la relación. El proyecto de investigación titulado Escuelas familiares: escenario para el encuentro de la Universidad de Caldas en Manizales, Colombia del cual se deriva el articulo que se presenta asume las escuelas familiares como:

Escenario de encuentro entre los(as) maestros(as), las familias, los(as) estudiantes y otros agentes educativos para reflexionar y dialogar sobre la realidad y el sentido de ser niño o niña en el contexto actual; identificar las condiciones de riesgo y posibilidad que se implican en su desarrollo y promover las capacidades de las familias, los maestros y maestras para compartir experiencias, formarse, identificar oportunidades de relación y construir conjuntamente alternativas que promuevan la titularidad de derechos, en otras palabras el desarrollo humano. (Rodríguez, 2014. p. 4)

El proyecto se inscribe en el Centro de Acompañamiento a las Familias ${ }^{1}$ (CAF), específicamente en su Programa de Escuelas Familiares, y el Departamento de Estudios de

\footnotetext{
'El Centro de Acompañamiento a las Familias se concibe como un escenario socioacadémico en el que convergen la investigación, la formación y la proyección en torno a las familias y sus integrantes. Se orienta a la articulación de prácticas académicas de la Facultad de Ciencias Jurídicas y Sociales en el que convergen los diferentes programas de pregrado y postgrado a través de su actuación en escenarios en los que confluyen:
} 
Familia de la Facultad de Ciencias Jurídicas y Sociales de la Universidad de Caldas. En términos académicos y para cumplir con los procesos de educación propios de la Investigación acción participante se vincularon estudiantes de prácticas de pregrado de los programas de Acción Física Humana, Diseño Visual, Desarrollo Familiar y Educación Física.

El objetivo general: Promover las escuelas familiares como estrategia de fortalecimiento de la relación familia y escuela y por esta vía el reconocimiento de los niños, niñas y adolescentes como sujetos de derechos. Sus objetivos específicos: a) Fortalecer la participación de las familias en la escuela como agentes corresponsables de los procesos educativos, y b) Fortalecer las capacidades protectoras de las familias y de las escuelas para la promoción de los vínculos de afecto, respeto, cuidado, solidaridad y confianza como pilares esenciales de la formación de los niños y las niñas.

Desde la perspectiva epistemológica, el proyecto se instaló en el constructivismo sociocultural y metodológicamente en la Investigación Acción Participante (IAP). El énfasis estuvo en el enfoque de las capacidades y el desarrollo humano.

\section{Metodología}

Cabe iniciar mencionando que el proyecto se orientó desde la Investigación Acción Participante (IAP) para lo cual se reconocieron dos procesos: el primero, conocer y reflexionar sobre la realidad vivida por la población en términos de la de la relación familia escuela y, el segundo, actuar para fomentar la participación de las familias en las escuelas y fortalecer sus capacidades educativas como familias y maestros(as). A la luz de la metodología, la educación fue la mediación para la reflexión con intención transformativa. El estudio se realizó en una institución educativa de básica primaria que incluye tres centros escolares en el sector rural de la ciudad de Manizales, Colombia. Se trabajó con 177 familias, 200 niños y niñas y 11 docentes.

Los momentos investigativos en función de la IAP y la intencionalidad del proyecto, sus técnicas e instrumentos y sus principales resultados fueron:

\section{A. Contextualización de la escuela familiar en el escenario escolar}

La contextualización se asume como un momento comprensivo de inserción y conocimiento de la institución y la comunidad educativa, en cuanto a sus características institucionales, sociopolíticas, económicas, culturales y ambientales; unido a las particularidades sociodemográficas que caracterizan tanto a las familias como a los(as) maestros(as). Las técnicas utilizadas fueron la cartografía social, el mapeo de actores y la caracterización sociodemográfica.

Entre los resultados: Las escuelas y las familias se ubican en sectores vulnerables de la ciudad (estrato socioeconómico uno²); maestros(as) con formación universitaria y edades

familias, grupos, comunidades e instituciones en orden a generar procesos de conocimiento, de transformación e incidencia en política pública.

${ }^{2}$ En Colombia la estratificación socioeconómica hace referencia a la clasificación de los inmuebles residenciales que deben recibir servicios públicos. El estrato socioeconómico uno corresponde a parte de la población que alberga usuarios con menores recursos. 
entre 25 y 45 años; familias con tipología nuclear, monoparental con jefatura femenina y extensas, con edades entre los o y 45 años; y niveles de educación formal de secundaria incompleta. Las familias no cuentan con los recursos y oportunidades materiales suficientes para atender los requerimientos de los niños y niñas.

\section{B. Diagnóstico Participativo}

Corresponde a la identificación, priorización y análisis comprensivo de las situaciones que vulneran o potencian el desarrollo de la población de trabajo. En lo particular del proyecto concierne a las situaciones que lesionan o promueven la condición de derecho de los niños y niñas y de los requerimientos y capacidades de sus familias y sus maestros(as) para responder a la situación. Así también de conocer la participación de las familias en los procesos escolares.

El proceso diagnóstico se realizó con cada uno de los grupos poblacionales -niños(as), familias y maestros(as) - a partir de cuatro ejes problémicos: niños(as) titulares de derechos, democratización de las relaciones familiares, niños(as) y ciberespacio, éxito y permanencia escolar. En cada uno de los ejes se incluía el reconocimiento de oportunidades y capacidades con las que contaban las familias y los maestros(as) para responder a las situaciones evidenciadas. Se utilizó la recuperación histórica con base en líneas de tiempo y narrativas para identificar la historia de vida familiar y escolar, comprender el presente y proyectar acciones futuras. El trabajo se complementó con la entrevista abierta, que involucraba indicadores de las propuestas de Sen (2000), Nussbaum (2012), Doyal y Gouhh (1994) y Hornby (2011).

Entre los factores de riesgo se identificaron prácticas familiares autoritarias que someten a niños(as) y mujeres a situaciones de violencia, inequidad de género y generación, pocos espacios de comunicación y ocio familiar, descalificación y desconocimiento afectivo en las relaciones, falta de claridad en la definición de normas y reglas, poca participación de la familia en la escuela y falta de implicación de la familia en los procesos escolares.

C. Elaboración y ejecución de planeas de acción o proyectos socioeducativos para la escuela familiar

La elaboración de planes de acción corresponde al diseño de propuestas de actuación para responder a las situaciones identificadas en el diagnóstico participativo. En otras palabras, representa la planeación del proceso para lograr las mejoras y las transformaciones necesarias para promover la titularidad de derecho de los niños-as. La ejecución atañe al proceso educativo realizado con la población y orientado hacia la reflexividad y el cambio.

A partir de los resultados del diagnóstico y retomando las intenciones y propósitos de la comunidad educativa escolar, el equipo de investigación incluidos los estudiantes practicantes formuló 10 proyectos socioeducativos orientados a las causas y no solo a los efectos inmediatos, que es lo que generalmente se espera, por ejemplo bullying, maltrato infantil, drogadicción, entre otras. En la búsqueda de prevención y promoción, retomando los planteamientos de Di Marco, Faur y Méndez (2005), se tiene en consideración la democratización de las relaciones como principio para la transformación "de estilos de vida 
en los modelos de género, de autoridad, y en la concepción de los derechos de la infancia junto con una concepción del cuidado mutuo entre todos los miembros del grupo familiar" (p.17) y de la escuela.

En términos de una visión de proceso y participación, los proyectos se ejecutaron en tres escenarios: formación docente, sociofamiliar y ocio y desarrollo educativo. El primero implicó el trabajo de formación con los(as) maestros(as). El segundo involucró a los niños(as) en las aulas de clase, en una unidad de aprendizaje del área de ciencias sociales denominada escuela familiar, y a sus familias en talleres de formación familiar o semilleros y "tertuliaderos" familiares. El tercero corresponde a encuentros de ocio donde, a través de la danza, el deporte, la lúdica y el arte, se encontraron los tres actores.

En la siguiente tabla se nombran los proyectos socioeducativos que orientaron las escuelas familiares y los escenarios de trabajo en los que se incluyeron:

Tabla 1.

Proyectos socioeducativos en escuelas familiares en Manizales, Colombia entre 2015 y 2018

\begin{tabular}{|c|c|c|c|}
\hline \multirow{2}{*}{$\begin{array}{l}\text { Institución } \\
\text { educativa }\end{array}$} & \multirow[b]{2}{*}{$\begin{array}{l}\text { Centro } \\
\text { escolar }\end{array}$} & \multicolumn{2}{|c|}{ Proyectos educativos } \\
\hline & & $\begin{array}{l}\text { Escenario Formación docente y } \\
\text { sociofamiliar }\end{array}$ & $\begin{array}{c}\text { Escenario Ocio y desarrollo } \\
\text { educativo }\end{array}$ \\
\hline \multirow{6}{*}{$\begin{array}{l}\text { José } \\
\text { Antonio } \\
\text { Galán }\end{array}$} & & $\begin{array}{l}\text { Escuela y familia, promotoras de } \\
\text { desarrollo humano de los niños y } \\
\text { niñas. }\end{array}$ & \\
\hline & Alto Bonito & $\begin{array}{l}\text { Familia y escuela, escenarios de } \\
\text { afecto: una estrategia para el } \\
\text { desarrollo integral de niños y niñas } \\
\text { como sujetos de derechos. }\end{array}$ & $\begin{array}{l}\text { La familia y la escuela, } \\
\text { promotoras de relaciones } \\
\text { democráticas a partir del teatro. }\end{array}$ \\
\hline & $\begin{array}{l}\text { Santa } \\
\text { Teresita del } \\
\text { Niño Jesús }\end{array}$ & $\begin{array}{l}\text { Los procesos de educación en el } \\
\text { escenario sociofamiliar, una } \\
\text { reflexión para la acción desde los } \\
\text { vínculos socioafectivos. }\end{array}$ & $\begin{array}{l}\text { El biodrama, una mirada hacia la } \\
\text { relación familia escuela. } \\
\text { El ocio como promotor de } \\
\text { vínculos socioafectivos en la }\end{array}$ \\
\hline & & $\begin{array}{l}\text { La construcción de relaciones } \\
\text { democráticas en la relación familia- } \\
\text { escuela. }\end{array}$ & $\begin{array}{l}\text { escuela y la familia, a través de } \\
\text { juegos predeportivos. }\end{array}$ \\
\hline & & $\begin{array}{l}\text { Educación afectiva, un compromiso } \\
\text { de la familia y la escuela. }\end{array}$ & $\begin{array}{l}\text { La relación familia-escuela a } \\
\text { través de la danza y el teatro. }\end{array}$ \\
\hline & $\begin{array}{l}\text { Bajo } \\
\text { Corinto }\end{array}$ & $\begin{array}{l}\text { La democratización de relaciones, } \\
\text { un espacio de participación familiar } \\
\text { que permite fortalecer la relación } \\
\text { familia-escuela. }\end{array}$ & \\
\hline
\end{tabular}

\section{Evaluación y sistematización}

Este momento -formativo (durante) y sumativo (final)- es una estrategia de análisis para reconocer los aprendizajes, los avances y limitaciones, y reconstruir con sentido la experiencia. Las técnicas fueron el taller, las entrevistas, los instrumentos, guías de observación y fichas de seguimiento. 
Los resultados de la evaluación y sistematización se agruparon en tres categorías: a) la escuela familiar, una oportunidad para la relación familia - escuela, b) la participación de las familias en la escuelas, c) las capacidades educativas fortalecidas y co-construidas por las familias y los (as) maestros(as), un reto para la educación de los niños(as). El proceso, como se identifica en los resultados, permitió significar a las familias por las relaciones y los vínculos construidos más allá de las relaciones de consanguinidad y las estructuras predeterminadas. Reconocer la escuela como escenario para la formación más que para la transmisión lineal de conocimientos formales y la importancia de una relación propositiva. Las familias, no todas por supuesto, han asumido un compromiso con la escuela el cual se identifica en acompañamiento en las deberes escolares, diálogo con los(as) maestros(as), motivación a sus niños(as) sobre el sentido de la escuelas y sus posibilidades para la formación; implicación en actividades de manutención entre otras. Los discursos de las familias y los(as) maestros(as) expresan fortalecimiento de conocimientos sobre su lugar educativo para la promoción del desarrollo de sus niños(as), así como prácticas orientadas al relacionamiento, el reconocimiento y la equidad.

Con la observación de los resultados se evidenció que los niños, las familias y los maestros adquirieron un saber educativo con base en la interacción y la relación intergeneracional, con una intencionalidad transformativa que se expresa en nuevos discursos sobre el sentido y el lugar de las familias, los requerimientos de diálogo entre familia y escuela y el reconocimiento de las relaciones democráticas como fundamento de una equidad de género tendiente al reconocimiento y la inclusión. Este proceso facilitó la recreación y resignificación de la cultura familiar y escolar por parte de la población de trabajo, quien ha elaborado códigos de saber a partir de su propia realidad.

\section{Discusión y conclusiones}

La estrategia de escuela familiar, una oportunidad para el desarrollo humano en la relación familia-escuela

La escuela familiar, tal como se reconoce en los resultados que se presentan en este escrito, y en perspectiva del desarrollo humano, es una oportunidad para que dos de los actores esenciales en la formación de los niños y las niñas se reconozcan como agentes educativos que deben complementarse desde el encuentro y la co-construcción. En otras palabras, la estrategia, siguiendo a Sen (2000), es un escenario de oportunidades para actuar, más allá de las discontinuidades que los pueden separar.

Así pues, pensada y actuada de acuerdo con una educación reflexiva y donde las lógicas residen en los sujetos y en los procesos, la escuela familiar es una oportunidad para que familias y maestros(as) logren un ambiente protector que le permita a los niños y niñas "mejores herramientas posibles para la construcción de su vida, disfrutando de oportunidades para desarrollar su capacidad individual en un entorno seguro y propicio" (Vertel y Cuervo, 2013, párr. 1). De este modo, la escuela familiar, como oportunidad, es un entorno protector para plantear las condiciones adecuadas para el crecimiento y desarrollo de los niños y niñas como seres individuales, únicos, dignos, valiosos y capaces de dar y recibir.

Dimensionar la formación como mediación en las propuestas de escuela familiar es una oportunidad para que docentes y familias se encuentren en el diálogo y reflexionen sobre 
sus realidades y se comprometan a propiciar acciones orientadas al cambio. En este escenario, el acompañamiento especializado para crear y recrear sus propuestas de trabajo, y por esta vía articularlos al Proyecto Educativo Institucional (PEI), garantiza la sostenibilidad y fortalecimiento de la relación. La formación de estos dos agentes, como lo expresa Mayolas y de Guevara Pascual (2016), es un deber pero también un derecho por la responsabilidad compartida.

En concordancia con lo expuesto hasta ahora, cabe destacar que las escuelas familiares pensadas en el diálogo y el encuentro son una oportunidad para que las familias compartan experiencias de vida y aprendan de otras experiencias. Encontrarse en la narrativa del otro(a) les ayuda a reconocer que no están solas y que las prácticas educativas que siguen con sus niños y niñas pueden ser comunes o diferentes. Se encuentran en los deseos, tensiones y temores de ser responsables del ser y el hacer ajeno.

Asimismo, la estrategia de escuela familiar entrega a las familias y docentes la oportunidad de reconocer a los niños y niñas como sujetos de derechos. Seres sensibles, afectivos, soñadores, capaces de tomar algunas decisiones y emitir juicios frente al mundo. La escuela, bajo la concepción de familia heterogénea, diversa e incluyente, como escenario de formación y construcción de conocimiento, y de la educación como proceso de mediación para la formación, rompe con prácticas tradicionales que recogen la obediencia y la sumisión. La oportunidad de vincularse permite, siguiendo a Galvis (2011), explorar la igualdad como principio atributivo que libera a los niños y niñas de la dependencia y los define como sujetos titulares de los derechos.

Finalmente, la escuela familiar fundamentada en el marco la alianza interinstitucional representa el conjunto de recursos y voluntades, también para actuar en función de responsabilidades, de alguna manera, compartidas. Igualmente, el trabajo en red y la interdisciplinariedad dotan de sentido la estrategia de escuela familiar y soportan a las familias y las escuelas para que se definan y accionen con un propósito común.

La participación de la familia en la escuela: una oportunidad, una capacidad.

La participación de las familias en la escuela se expresa como una forma de relacionamiento con el centro escolar y deriva un compromiso con la educación de sus niños(as). De esta manera, la participación no es una actuación ingenua sino una expresión simbiótica dotada de sentido en la que el maestro(a) igual tiene un compromiso de promoverla, asumirla y respetarla. Es decir, es un asunto de dos para cumplir con un objetivo común; no se puede esperar que las familias lo hagan en solitario. en palabras de Blanco, es "un rasgo de una educación que refleje calidad y equidad y destaque el esfuerzo compartido por parte de la comunidad educativa" (2014, p.18).

En tanto oportunidad, la participación de las familias en los centros escolares se constituye en una acción pedagógica que en palabras de Moya se define como "una situación formadora" (2002, p. 20) orientada, en este caso, al desarrollo de los niños(as); al tiempo en una mediación para la relación entre los actores que se implican en su proceso educativo. De esta manera, recogiendo los planteamientos de Villalobos Martínez (2017) y García Sanz, Hernández Prados, Parra Martínez y Gomariz Vicente (2016, p.99), a través de la participación se crea una oportunidad de diálogo favorable que además de permitir unas relaciones amables favorece la implicación conjunta en la gestión educativa. 
En tanto capacidad, la participación de las familias en la escuela, como lo expresan Meza Rueda y Páez Martínez (2016), les permite hacer parte -actuar- de los procesos escolares, involucrarse en la planeación institucional y co-crear con los(as) maestros(as) alternativas para fortalecer sus conocimientos, revisar sus fines educativos y ajustar o cambiar los discursos y las prácticas a favor de ambientes socioafectivos familiares caracterizados por relaciones democráticas

\section{Escuelas familiares y desarrollo humano, una lectura a partir del fortalecimiento de las capacidades}

Las capacidades son "las diversas cosas que una persona puede valorar, hacer o ser" (Sen, 2000, p. 99), y "un conjunto de oportunidades (habitualmente interrelacionadas) para elegir y actuar" hacen posible en el contexto de la relación familia - escuelas que los grupos familiares y los-as maestros-as puedan fortalecer y ejercer su agencia en función de la educación de niños y niñas.

Si bien el fortalecimiento de capacidades para la formación de esta población es una de las responsabilidades de las familias y los(as) maestros(as), hay que reconocer que no es una tarea del todo fácil. De un lado, ser y hacer familia es un asunto posible, pero al tiempo complejo si se tiene en cuenta que en este proceso se involucran subjetividades, contextos y relaciones, en los cuales las familias asumen sus compromisos educativos desde lo cotidiano, sin recibir, como lo dice Rodríguez (2018a), ninguna preparación pero sí, la mayoría de las veces, con la mejor intención. Además, es claro que este grupo social...

Se encuentra en un proceso de cambio, relacionado con los nuevos lugares sociales de sus integrantes y con un contexto de transformación social que trae consigo nuevos estilos de vida y nuevas formas de organización y de relación que invitan a reconocer la existencia de una creciente multiplicidad y variedad de acuerdos de vida familiar. (Rodríguez, 2013, p. 54)

De otro lado, ser y hacer maestro involucra objetivos y tareas que, aunque apasionantes por lo que representan en la constitución humana, en ocasiones no logran alcanzarse por razones de contexto, de política y de condición que no favorecen propósitos de humanización, y sí, por el contrario, se sitúan en lo funcional escolar. Los docentes podrán tener las mejores intenciones, pero muchas veces no cuentan con los recursos o posibilidades para complementar su formación.

Por todo esto, las familias y los(as) maestros(as), parafraseando a Rodríguez (2018b), deben potenciarse a sí mismos en su condición de protección y promoción de los derechos de los niños y niñas mediante la consolidación de sus propias capacidades en función de la educación de los otros. Para ello, deben asumir el reto, no solo del diálogo, sino también de la formación que les permita, en términos de las capacidades, hacer y ser (Sen, 2000) y reconocer las oportunidades de las que disponen para elegir y actuar (Nussbaum, 2012) como educadores y a favor de sus estudiantes.

Así, las experiencias de escuelas familiares que derivan esta reflexión, co-construidas desde la praxis y con intencionalidad transformativa más allá de la funcionalidad y la capacitación, dimensionan una propuesta de desarrollo humano situada en el fortalecimiento de seis de las diez capacidades propuestas por Nussbaum.

1. Con respecto a la cognición. Emerge la capacidad de imaginar, pensar y razonar sobre el sentido de ser niño y niña hoy, de los requerimientos que se implican en la titularidad de sus 
derechos, de los riesgos propios que le enfrenta el contexto y de buscar, desde la prevención, alternativas para enfrentarlos. Así también, se expresa la cognición cuando se comprende familia en la diversidad y la heterogeneidad, en el requerimiento de unas relaciones democráticas sustentadas en la "necesidad de promover vínculos entre hombres y mujeres, en los que se respeten las diferencias de cada uno o cada una, para que estas no se conviertan en motivos que justifiquen la desigualdad y la subordinación" (Di Marco, Faur y Méndez, 2005 p. 17).

Igualmente, la cognición surge cuando los participantes de las escuelas familiares piensan y asumen la estrategia como una propuesta socioeducativa que, recogiendo los planteamientos de Nussbaum (2012) sobre capacidades, es "adecuada" para el aprendizaje y la apropiación de contenidos que han sido configurados desde la experiencia cotidiana, complementados y articulados con discursos teóricos que, unidos, hacen posible la reflexividad que les implica volver sobre sus prácticas de vida y pensar cómo transformarlas.

2. Con respecto a las emociones. La construcción de vínculos afectivos promovidos desde las escuelas familiares se convierte en una potencialidad que dimensiona la condición humana de los niños y niñas; asimismo, en una mediación pedagógica que, de alguna manera, aporta a su desarrollo físico, cognitivo y emocional y posibilita, no solo su autovaloración, sino la posibilidad de entretejer relaciones cálidas y receptivas; además, de animar el interés por el conocimiento, despertar la creatividad, la sensibilidad, privilegiar una actitud positiva y vivenciar los derechos como una forma de reconocimiento de sí mismo y del otro.

3. Con respecto a la razón práctica. A través de las escuelas familiares, de acuerdo con los planteamientos de Nussbaum (2012), las familias y los(as) maestros(as) se vinculan en reflexiones críticas donde logran generar concepciones sobre el sentido del "bien" para los niños y niñas y del "bien" de ser actores fundantes en la formación. De esta manera, familias y maestros(as) son libres e iguales (Rawls, 1996, p. 338) gracias a que tienen la capacidad para hacerse su propia concepción del bien en función de los sujetos que son el eslabón de su relación. Las escuelas familiares en función de la razón práctica y siguiendo los planteamientos de Aguirre (2016) ayuda a las familias que no se conforman con educar a sus hijos, sino que lo quieren hacer mejor. En este orden, la formación recibida en la escuela familiar les permite aprendizajes para gestionar las relaciones en sus familias teniendo claridad de cómo actuar ante determinadas situaciones; analizar los conflictos y los procesos de vida con sus niños-as y así fortalecer sus capacidades comunicativas, por ejemplo, para responder a su función parental.

4. Con respecto a la afiliación. La escuela familiar permite el relacionamiento entre familias y maestros(as), haciendo posible conjugar propósitos educativos. En la relación se reconocen intereses comunes y se comprometen diversas formas de interacción. Así también, la afiliación en el contexto de la escuela familiar incluye el sentido de la participación y la corresponsabilidad (Sánchez y Facal , 2018; Galián, García y Belmonte2018; Martínez, 2010; y Vila, 2003), de un lado, de la familia en el escenario escolar y, de otro, de la escuela de reconocerla como agente activo del desarrollo. Se trata entonces de compromiso, no delegación ni apoyo. 
5. Con respecto al humor y al juego. Los tiempos de las familias y los maestros, la gran mayoría de las veces, son opuestos y generan tensión porque difícilmente coinciden. Es necesario, como lo muestra la experiencia objeto de esta reflexión, que las escuelas familiares consideren el ocio como una mediación para el aprendizaje y para el encuentro donde los participantes puedan reír y disfrutar desde la comprensión los procesos en los que se involucran. En perspectiva de Cuenca (2011), hacer ocio promueve el desarrollo humano (p. 28). De esta manera, el ocio es un evento que proporciona conocimiento como resultado de haber sentido y disfrutado la experiencia.

6. Con respecto a la individualidad. Esta capacidad, en términos de la propuesta de escuelas familiares orientada al desarrollo humano, se sitúa en el reconocimiento de familia y maestro como agentes capaces de incrementar la dimensión de referenciación del ser, en este caso los niños y niñas. Del mismo modo, la individualidad en vista de la potenciación de estos últimos como sujetos constructores de su saber y responsables de su vida y por tanto de su aprendizaje. En virtud de lo anterior, agente es "la persona que actúa y provoca cambios y cuyos logros pueden juzgarse en función de sus propios valores y objetivos, independientemente de que los evaluemos o no también en función de algunos criterios externos" (Sen, 2000, p. 35).

\section{Referencias}

Aguirre, A.M. (2016) Escuela de Padres. En Aguirre, A.M., Caro, C., Fernández, S. y Silvero, M. (2016). Familia, escuela y sociedad para maestros. España: Universidad Internacional de la Rioja, UNIR

Anderson, G. L. (2002). Hacia una participación auténtica: deconstrucción de los discursos de las reformas participativas en educación. En M. Narodowski, M. Nores y M. Andrada (Coords.), Nuevas tendencias en políticas educativas. Estado, mercado y escuela (pp. 145-200). Buenos Aires, Argentina: Ediciones Juan Granica.

Arias, B.E. La infancia como sujeto de derechos. un análisis critico. Revista Ratio Juris Vol. 12 No 24 (enero-junio 2017) pp. 127-142

Blanco, M.V. (2014). Una propuesta de trabajo para implicar a las familias en las escuelas de E.I. España: Universidad de Cádiz.

Cuenca, M. (2011). El ocio como ámbito de Educación Social. Educación social: Revista de intervención socioeducativa, 47, 25-40.

Di Marco, G. Faur, E. y Méndez (2005). Democratización de las familias. Buenos Aires, Argentina. UNICEF

Doyal, L. y Gouhh, A. (1994). A Theory of Human Need. New York: The Guilford Press

Galián, N., García, M. P. y Belmonte, M. L. (2018). Evaluación de la participación familiar en el proceso educativo de los discentes desde la percepción del profesorado. Revista Electrónica Interuniversitaria de Formación del Profesorado, 21(3), 45-62.

Galvis, L. (2011). Pensar la familia de hoy. El paradigma de los derechos humanos. Fin del régimen patriarcal. Bogotá, Colombia: Ediciones Aurora.

García Sanz, M.P., Hernández Prados, M.A., Parra Martínez, J., Gomariz Vicente, M.A. (2016). Participación familiar en la etapa de educación primaria. Perfiles educativos, 38(154), 97-117. 
Giroux, H. A. (2006). La escuela y la lucha por la ciudadanía. Ciudad de México, México: Siglo XXI Editores.

Hornby, G. (2011). Parental involvement in childhood education: building effective schoolfamily partnerships. New York: Springer.

Lacasa, P. (1997). Familias y escuelas. Caminos de la orientación educativa. Madrid: Visor.

H Gómez Buendía - Hacia un desarrollo humano. Programa de Naciones Unidas para el Desarrollo. PNUD. TM Editores, 1998.

Martínez, S. D. (2010). La educación, cosa de dos: La escuela y la familia. Revista digital para profesionales de la enseñanza, 1(8), 1-15.

Mayolas, A. A. y de Guevara Pascual, B. L. (2016). Análisis de la escuela y la familia: ideas para compartir el proceso educativo. REXE. Revista de Estudios y Experiencias en Educación, 15(28), 197-208

Meza Rueda, J, L. y Páez Martínez, R. (2016). Familia, escuela y desarrollo humano. Rutas de investigación educativa. Colombia. Universidad de La Salle, CLACSO.

Nussbaum, M. C. (2012). Crear capacidades. Propuesta para el desarrollo humano. Barcelona, España: Paidós.

Páez, R. M. y Pérez, N. (2018). Educación familiar. Investigación en contextos escolares. Buenos Aires, Argentina: CLACSO.

Rawls, J. (1996). El liberalismo político. Barcelona, España: Crítica.

Rodríguez, Z.E. (2018a). Qué y cómo se enseña y aprende en la familia. Un asunto de interés para la escuela. Revista Latinoamericana de Estudios Educativos, 14(2), 132-157.

Rodríguez, Z.E. (2018b). La educación familiar, un asunto de la vida en familia y de interés teórico. En R. M. Páez y N. Pérez, Educación familiar. Investigación en contextos escolares. (pp. 21-51) Buenos Aires, Argentina: CLACSO.

Rodríguez, Z.E. (2014). Escuelas familiares. Escenarios para el encuentro. Departamento de Estudios de Familia, Vicerrectoría de Proyección. Universidad de Caldas.

Rodríguez, Z.E. (2013). Prácticas educativas familiares en la familia en situación de transnacionalidad. Análisis de la interactividad y la influencia educativa (tesis doctoral). Manizales, Colombia: Rudecolombia - Universidad de Caldas.

Rogoff, B. (1997). Los tres planos de la actividad sociocultural: apropiación participativa, participación guiada y aprendizaje. En J. Wertsch, P. del Río y A. Álvarez (Eds.), La mente sociocultural. Aproximaciones teóricas y aplicadas (pp. 111-128). Madrid, España: Fundación Infancia y Aprendizaje.

Sánchez, N. y Facal, D. (2018). Las relaciones entre las familias y la escuela en la etapa adolescente: implicaciones para el Departamento de Orientación. Revista Electrónica Interuniversitaria de Formación del Profesorado, 21(3), 63-74.

Sen, A. (2000). Desarrollo y libertad. Buenos Aires, Argentina: Planeta.

Tedesco, J. C. (1995). El nuevo pacto educativo. Educación, competitividad y ciudadanía en la sociedad moderna. Madrid, España: Anaya.

Vertel, L. y Cuervo, L. (4 de noviembre de 2013). ¡A fortalecer ambientes protectores para la niñez!. Crianza y salud para el bienestar de la familia. Recuperado de: https://crianzaysalud.com.co/a-fortalecer-ambientes-protectores-para-la-ninez/

Vila, I. (2003). Familia y escuela: dos contextos y un solo niño. En La participación de los padres y madres en la escuela (pp. 27-38). Barcelona, España: Graó. 